\title{
Revision of COVID in China: a thorough analysis of epidemic situations and Chinese strategies during the pandemic
}

\author{
Yawen Deng ${ }^{1, \dagger}$, Yushun $\operatorname{Tian}^{2, \dagger}$, Yintong Wang ${ }^{3, \dagger}$ and Yuan Xing ${ }^{4, \dagger, *}$ \\ ${ }^{1}$ The High School Attached to Jiangxi Normal University Sino-U.S., Jiangxi, China \\ ${ }^{2}$ Walt Whitman High School, Maryland, the United States \\ ${ }^{3}$ University of North Carolina at Chapel Hill, North Carolina, the United States \\ ${ }^{4}$ Shanghai Pinghe School, Shanghai, China \\ ${ }^{\dagger}$ These authors contributed equally to this paper.
}

\begin{abstract}
COVID-19, the disease caused by SARS-CoV-2, emerged in December 2019 in Wuhan, China, when Chinese authorities confirmed several patients with fever, difficulty breathing, and invasive lesions on the lungs. The virus then spread quickly across the globe, and by May 17, there were a total of 18,151,717 cases worldwide. While some countries still have an exponentially increasing rate of new cases, China has stabilized the spread of the virus. In the review of COVID-19 in this paper, we used sources from Google Scholar, PubMed, Research Gate, etc. In addition, when investigating public policy data and the number of infections, we also used the statements and relevant data from the official websites of the governments of Dalian and Beijing. Our investigation is based on the summary and analysis of the above data to reach our conclusion. We investigated the basic characteristics of COVID-19, the corresponding Sinovac vaccine and mRNA vaccine, the first round of outbreaks in Wuhan, Beijing, and Dalian, and the warning role of COVID19 in the future. We demonstrated that keeping social distance and isolating infected patients in time can effectively block the transmission of the virus. This paper exposes the necessary public health measures during the pandemic, providing experiences and lessons for a potential future pandemic.
\end{abstract}

\section{Introduction}

COVID-19 is the disease caused by SARS-CoV-2, the coronavirus that emerged in December 2019. Broadly, as a respiratory virus, COVID-19 causes breathlessness, fatigue, and muscle ache. As the pandemic has evolved and documented clinical case histories have accumulated, a new symptom began to emerge - the partial or total loss of the sense of taste and smell. This is not unusual for a respiratory viral infection, but what was unique is that people had this symptom without any other usual infection symptoms. It's now clear that the coronavirus doesn't just attack the respiratory system, and some people have reported gut issues and problems with their kidneys. Covid-19 first emerged in mid-December 2019 in Wuhan, China, when Chinese authorities confirmed several patients with fever, difficulty in breathing, and invasive lesions on the lungs. The virus then spread quickly across the globe and was then announced as a pandemic by WHO. By May 17, there were a total of $18,151,717$ cases worldwide, with $99.4 \%$ patients in mild condition and $0.6 \%$ patients in serious condition. There are reported cases across 221 countries. Some countries have stabilized the spread of a virus like China, while some still have an exponentially increasing rate of new cases. A variety of strategies have been implemented in different countries in the face of the pandemic. The purpose of this review paper is to provide the basic framework of how the pandemic originated and Chinese strategies fighting against the pandemic, providing lessons and examples on dealing with public health emergency to reduce financial impact and better protect global citizens in the future. To this end, we present a thorough review of the COVID 19 and the strategies utilized during the pandemic in China. The rest of this paper is organized as follows. In part 1 of the main body, basic information on the virus was discussed. In part 2 of the main body, we presented a revision for $1^{\text {st }}$ round pandemic centered in Wuhan. In part 3 of the main body, the epidemic outbreak in Beijing, Dalian, and Shijiazhuang were analyzed. In the last part, comparison, characteristics, regulation, and referential from different periods were discussed.

\section{Introduction for covid-19}

\subsection{Epidemiology and symptoms of COVID-19}

In 2020, a newly identified coronavirus, SARS-COV-2, caused a worldwide pandemic of a respiratory illness called severe acute respiratory syndrome coronavirus disease 2019 (COVID-19).

\footnotetext{
*Corresponding author: 167922@mcpsmd.net
} 
Covid-19 first emerged in mid-December 2019 in Wuhan, China, when Chinese authorities confirmed several patients with fever, difficulty in breathing, and invasive lesions on the lung [1]. By January 2020, 41 patients were laboratory confirmed as having infection of COV-19 in Wuhan. Among them, $49 \%$ were found to be at the age of $25-49$, and about $34 \%$ of the patients aged 50-64 years old. 13 of the 41 patients were admitted to ICU because of the high-level oxygen support they needed. No more than half of them had a disease like diabetes $(20 \%)$, hypertension (14\%), cardiovascular disease $(15 \%)$, chronic obstructive pulmonary disease ( $2 \%)$, and chronic liver disease $(2 \%)$, as shown on table 1 [2].

Table 1. Characteristics of first-round patients in Wuhan [2]

\begin{tabular}{|c|c|c|c|c|}
\hline Characteristics & $\begin{array}{l}\text { All patients } \\
(n=41)\end{array}$ & $\begin{array}{l}\text { ICU care } \\
(n=13)\end{array}$ & $\begin{array}{l}\text { No ICU } \\
\text { care } \\
(n=28)\end{array}$ & \\
\hline Age, years & $\begin{array}{l}49.0 \quad(41.0- \\
58.0)\end{array}$ & $\begin{array}{l}49.0 \\
(41.0- \\
61.0)\end{array}$ & $\begin{array}{l}49.0 \\
(41.0-- \\
57.5)\end{array}$ & 0.6 \\
\hline Sex & $\ldots$ & $\ldots$ & $\ldots$ & 0.24 \\
\hline Man & $30(73 \%)$ & $1(85 \%)$ & $19(68 \%)$ & $\ldots$ \\
\hline Women & $11(27 \%)$ & $2(15 \%)$ & $9(32 \%)$ & $\ldots$ \\
\hline $\begin{array}{l}\text { Huanan Seafood } \\
\text { Market Exposure }\end{array}$ & $27(66 \%)$ & $9(69 \%)$ & $18(64 \%)$ & 0.75 \\
\hline Current Smoking & $3(7 \%)$ & 0 & $3(11 \%)$ & 0.31 \\
\hline Any comorbidity & $13(32 \%)$ & $5(38 \%)$ & $8(29 \%)$ & 0.53 \\
\hline Diabetes & $8(20 \%)$ & $1(8 \%)$ & $7(25 \%)$ & 0.16 \\
\hline Hypertension & $6(15 \%)$ & $2(15 \%)$ & $4(14 \%)$ & 0.93 \\
\hline $\begin{array}{l}\text { Cardiovascular } \\
\text { disease }\end{array}$ & $6(15 \%)$ & $3(23 \%)$ & $3(11 \%)$ & 0.32 \\
\hline $\begin{array}{l}\text { Chronic obstructive } \\
\text { pulmonary disease }\end{array}$ & $1(2 \%)$ & $1(8 \%)$ & 0 & 0.14 \\
\hline Malignancy & $1(2 \%)$ & 0 & $1(4 \%)$ & 0.49 \\
\hline Chronic liver disease & $1(2 \%)$ & 0 & $1(4 \%)$ & 0.68 \\
\hline
\end{tabular}

On January 9, the Chinese expert team released the pathological information of the virus and determined preliminarily that a new coronavirus was the cause.[3] Until May 31, 2020, a total of 83017 cases had been confirmed in China mainland. Among them, 78037 infected were cured and discharged from the hospital, whereas 4634 people died, indicating a cure rate of $94.3 \%$ and a fatality rate of 5.6 [3].

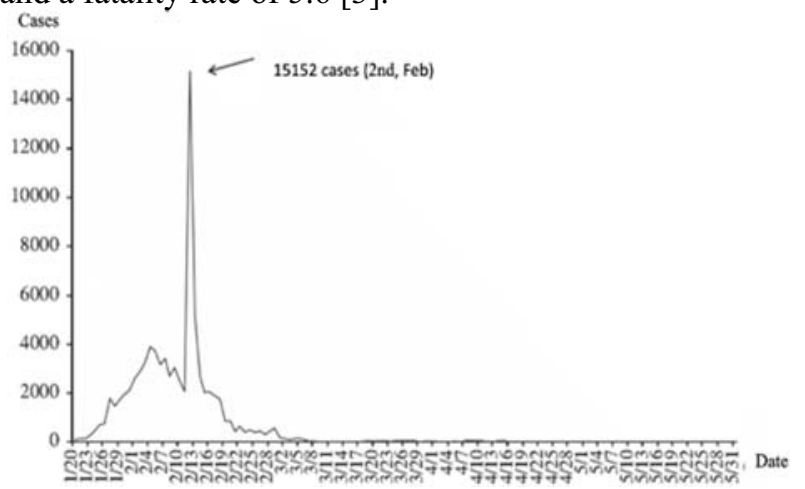

Figure 1. Confirmed cases variation along time [3].

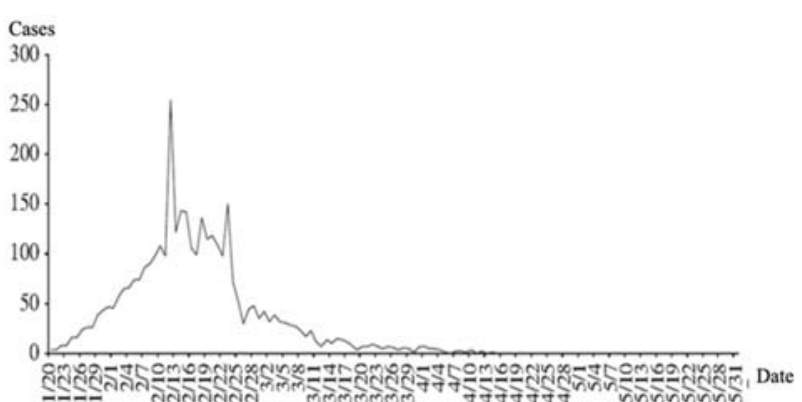

Figure 2. Confirmed case of death along time [3].

By May 17, there were a total of 18,151,717 cases worldwide, with $99.4 \%$ patients in mild condition and $0.6 \%$ patients in serious condition. There are reported cases across 221 countries, and some countries still have an exponentially increasing rate of new cases [4].

Chinese authorities have confirmed from the transmission with a household that the virus can spread from person to person [5]. The rate of secondary attack of SARS-CoV-2 in the household is around $16.3 \%$. Risk factors of transmission include how close family members interact and spousal relationships [6]. According to WHO, infected people spread the virus by small liquid particles, whose sizes range from large droplets to small aerosols, when they cough, sneeze or speak. Uninfected people can get infected when droplet-containing viruses get into their mouth, nose, or eyes by various means. For example, after infected people sneeze or cough on the surface of an object, other people may get infected by touching their eyes or nose after touching the contaminated surfaces. People who are in close contact with infected people are more likely to become infected [7]. It is also suggested that infection can happen during the incubation period of the patients [8].

When a person is infected with the virus, symptoms take between 5 and 6 days on average to appear but can take up to 14 days. The virus affects different people in different ways. Most infected people have mild or moderate symptoms and get better on their own. Thus, they could treat their symptoms at home [9]. However, about 1 in 6 will have severe problems like shortness of breath or dyspnea, chest pain, loss of speech or movement, and need to be hospitalized [10]. The most frequent symptoms, according to WHO, include fever, dry cough, and fatigue. Less frequent symptoms include aches and pains, sore throat, diarrhea, headache, loss of taste or smell, skin rash or discoloration of fingers or toes, etc [11]

Researchers have found that people with bone marrow cancer, organ transplant, severe lung and kidney conditions, who treat themselves with ACE2-increasing drugs, are at higher risk for severe COVID-19 infection. $[12,13]$.

At the same time, the elderly, minority populations, people with disabilities also suffer from higher vulnerability to COVID-19 infection [14].

\subsection{Pathology of SARS-COV-2}

Coronaviruses (CoVs) are a group of diverse and singlestranded RNA viruses [15]. There have been two novels, CoVs, severe acute respiratory syndrome CoV (SARS- 
$\mathrm{CoV}$ ) and Middle East respiratory syndrome $\mathrm{CoV}$ (MERS-CoV), emerged in human history [16].

SARs-CoV belongs to the family Coronaviridae under the order Nidovirales. The common symptoms caused by members of the family Coronaviridae include respiratory and intestinal infections [17]. SARS was known to be caused by SARs-CoV. SARS was first found in 2002 in Guangdong Province when an outbreak of pneumonia syndrome was noticed at the community level. During the pandemic in 2003, 8-96 cases and 774 deaths occur across five continents [17].

MERS was first isolated from a patient in Jeddah, Saudi Arabia [17]. The patient died because of severe pneumonia and organ failure in 2012. MARS-CoV has affected more than 1000 patients across the globe ever since 2012 are related to the Middle East symptom.

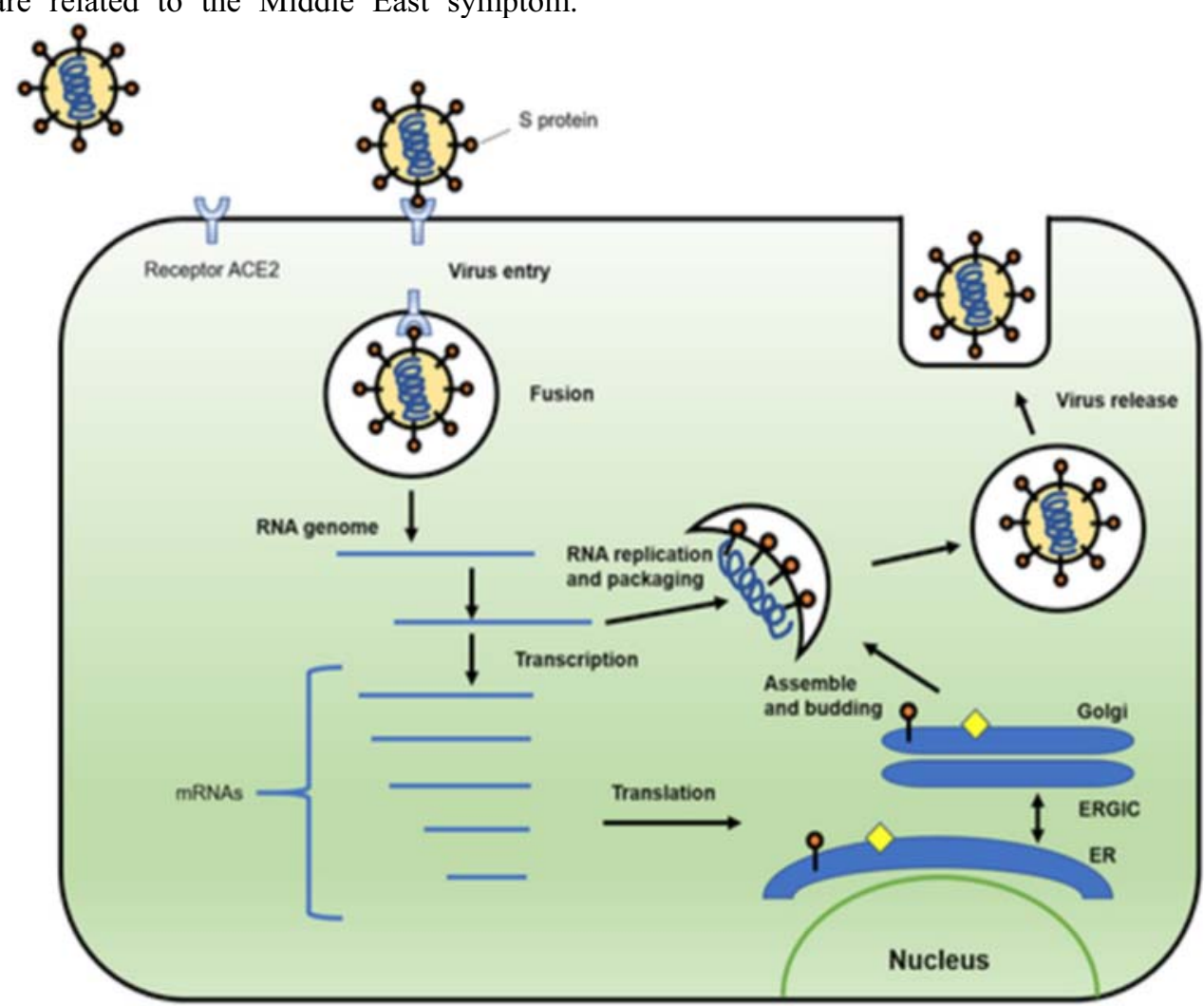

Figure 3. How the pathogen invades human cells [19].

\subsection{Current treating options for COVID-19}

So far, there are no specific antiviral drugs against COVID-19 infection. However, there are hundreds of clinical trials around the globe focusing on testing the existing drugs. According to the National Library of Medicine, the tested drugs include Dexamethasone, Duvelisib, Pulmozyme, Nafamostat Mesilate, etc. [21].

So far, according to the National Health Commission of the People's Republic of China, IFN- alpha and lopinavir/ritonavir are recommended as antiviral therapy [22]. The main function of the IFN-alpha 1 is to alert the organism in case of viral infection and inhibit virus multiplication [23]. Lopinavir is used against HIV infection as a fixed-dose combination with another protease inhibitor, ritonavir (lopinavir/ritonavir) [24].

There is also research proving remdesivir as an antiviral therapy as beneficial in the treatment of Covid-
Patients with SARS or MERS have symptoms like mild respiratory illness [18].

The pathogen that causes COVID-19 is named SARSCoV-2 (also known as 2019-nCoV). SARS-CoV-2 share some common features with the family of $\mathrm{CoV}$, but it also has its own distinct characteristics. For example, the cell receptor of the SARS-CoV-2 is ACE2 compared to DPP4 for MERS-CoV [19]. Studies suggested that SARS-CoV2 share about $79.5 \%$ similarity with SARS-CoV and reveals that it falls into Sarbecovirus of genus Betacoronavirus [20].

SARS-CoV-2 uses ACE2 as an entry receptor in ACE2-expressing cells. It binds to the receptor on the cell membrane, fuses with the membrane and enters the cell, as shown in figure 3 .
19. In the study, 541 of 1062 patients were assigned to the remdesivir group, and the remaining 521 patients were assigned to the placebo group. Among the patients in the experimental group, 531 of them received the assigned treatment, and the other 10 patients withdrew from the experiment. About 52 patients are given remdesivir treatment discontinuously before day 10 because of the adverse effect. Among the people receiving placebo, besides those who showed adverse effects, 517 people received placebo as assigned. The experiment showed that patients in the remdesivir group had a shorter time to recovery than patients in the placebo group, proving the effectiveness of treatment remdesivir [25]. Moreover, another research showed that Remdesivir could effectively reduce Middle East Respiratory Syndrome symptoms by improving lung damage [26].

In addition, some Traditional Chinese Medicines may play a role in the therapy of COVID-19. According to the research, Shufeng Jiedu Capsule (SFJDC), a traditional 
Chinese medicine (TCM) consisting of eight medicinal herbs combined with oseltamivir treatment, could significantly attenuate IAV-induced airway inflammation [27]. However, the effectiveness and safety of the drug still need to be confirmed by further experiments.

It always takes years to develop a specific drug for a certain disease. Concerning COVID-19, there is still a need to establish animal experiments to develop COVID19 specific drugs. There are experiments where scientists work to develop a primate model to study the COVID-19 virus and the possible drugs and vaccines.

\subsection{Vaccines}

A vaccine provides active acquired immunity to a particular infectious disease like COVID-19. Vaccines save millions of lives each year by training the body's immune system to recognize and fight against viruses. Scientists across the globe are collaborating on the treatment and vaccines for COVID-19. According to WHO, by February 2021, at least seven different vaccines have been used in countries. Moreover, there are more than 200 additional vaccines in development [28]. Several of the most advanced candidates that are on clinical trial include mRNA-1273 from Moderna, Ad5-nCoV from CanSino Biologicals, INO-4800 from Inovio, pathogenspecific aAPC from Shenzhen Geno-Immune Medical Institute [29].

In China, the Sinopharm, Sinovac vaccines are leading the way. Sinivac vaccine is an inactivated vaccine. It works by using killed viral particles to expose the body's immune system to the virus.

In China, the most promising vaccine currently in human trials is CoronaVac. The Beijing-based biopharmaceutical company Sinovac is behind CoronaVac. CoronaVac is currently in Phase 3 trial in Brazil. CoronaVac is an inactivated vaccine. It is an inactivated vaccine made of inactivating pathogens. Viruses are inactivated by heat or chemicals during the development of vaccines so that they are unable to cause disease in the human body. It works by exposing the killed viral particles in front of the body's immune system, producing memory cells that will react more effectively next time exposed to the real pathogen [30]. Some other inactivated vaccines developed in other counties include the United Kingdom's Oxford/AstraZeneca vaccine and the United States' Moderna vaccine. However, Oxford and Moderna use a small part of the SARS-CoV-2 virusthe spike protein whereas CoronaVac uses the whole, inactivated virus [30].

There are also mRNA vaccines for covid-19. One of the mRNA vaccines that has proven effective in preventing COVID-19 is Moderna's COVID-19 vaccine (mRNA-1273 vaccine). It is a lipid nanoparticle encapsulated mRNA vaccine. mRNA vaccine 1273 encodes the spike protein of Covid-19, causing the virus SARS-CoV-2 [31].

mRNA vaccines are more controllable during production compared with inactivated viruses so that the possibility of contamination can be reduced [31].

Although mRNA vaccines are a newly emerging technology and there is no approved product so far on the market, the speed of mRNA vaccine development against SARS-CoV-2 has been unprecedented globally [31].

\section{Pandemic revision- Wuhan}

\subsection{Time-line}

On December 8, 2019, the first case of unexplained pneumonia was officially reported. On December 31, 2019, Wuhan municipal government issued the first public notice on the novel coronavirus [31]. On January 3, 2020, the Wuhan government reported the new coronavirus for the second time, stating that it was still viral pneumonia. The number of patients increased to 44 , including 11 severe cases. For the first time, it was mentioned that the work of tracing the cause of disease began, and the common respiratory diseases such as influenza, avian influenza, and adenovirus infection were excluded. On January 9, 2020, the pathogen was identified as "coronavirus". Before and after January 10, 2020 , the number of beds in local hospitals in Wuhan is full, the number of "suspected patients" increases sharply, and some doctors and nurses are infected. On January 21, 2020, academician Zhong Nanshan told CCTV news broadcast that New Coronavirus pneumonia was a confirmed human successor; Wuhan implemented import and export control. On January 23, 2020, Wuhan officially implemented the measures of "blocking the epidemic areas of class A infectious diseases" stipulated in the "law on the prevention and control of infectious diseases", and entered into a state of city closure; public transportation such as public transportation, subway, and aircraft stopped operation. At the same time, Wuhan announced establishing a special hospital with a capacity of 1000 beds based on the model of Beijing Xiaotangshan Hospital, which is planned to be completed within 6 days. The first three new "shelter hospitals" in Wuhan were completed on February 5. On April 8, 2020, the closure of Wuhan ended, which took 76 days. On April 26, Wuhan was cleared in patients with COVID-19.

\subsection{Strategies adopted by Wuhan}

Wuhan has taken many epidemic prevention measures that can be used for reference during the COVID-19 epidemic, such as home isolation and shelter hospital. 


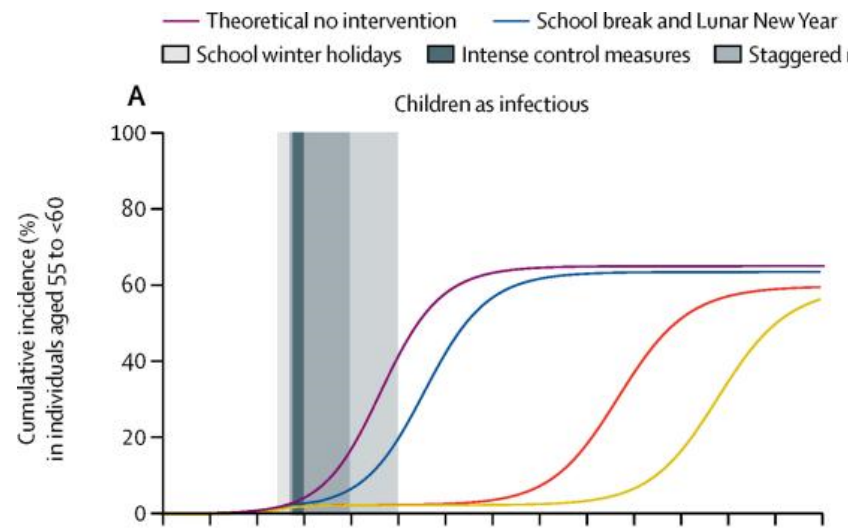

- Relax intervention in March - Relax intervention in April $\square$ School winter holidays $\square$ Intense control measures $\square$ Staggered relax of interventions (March) $\square$ Staggered relax of interventions (April)
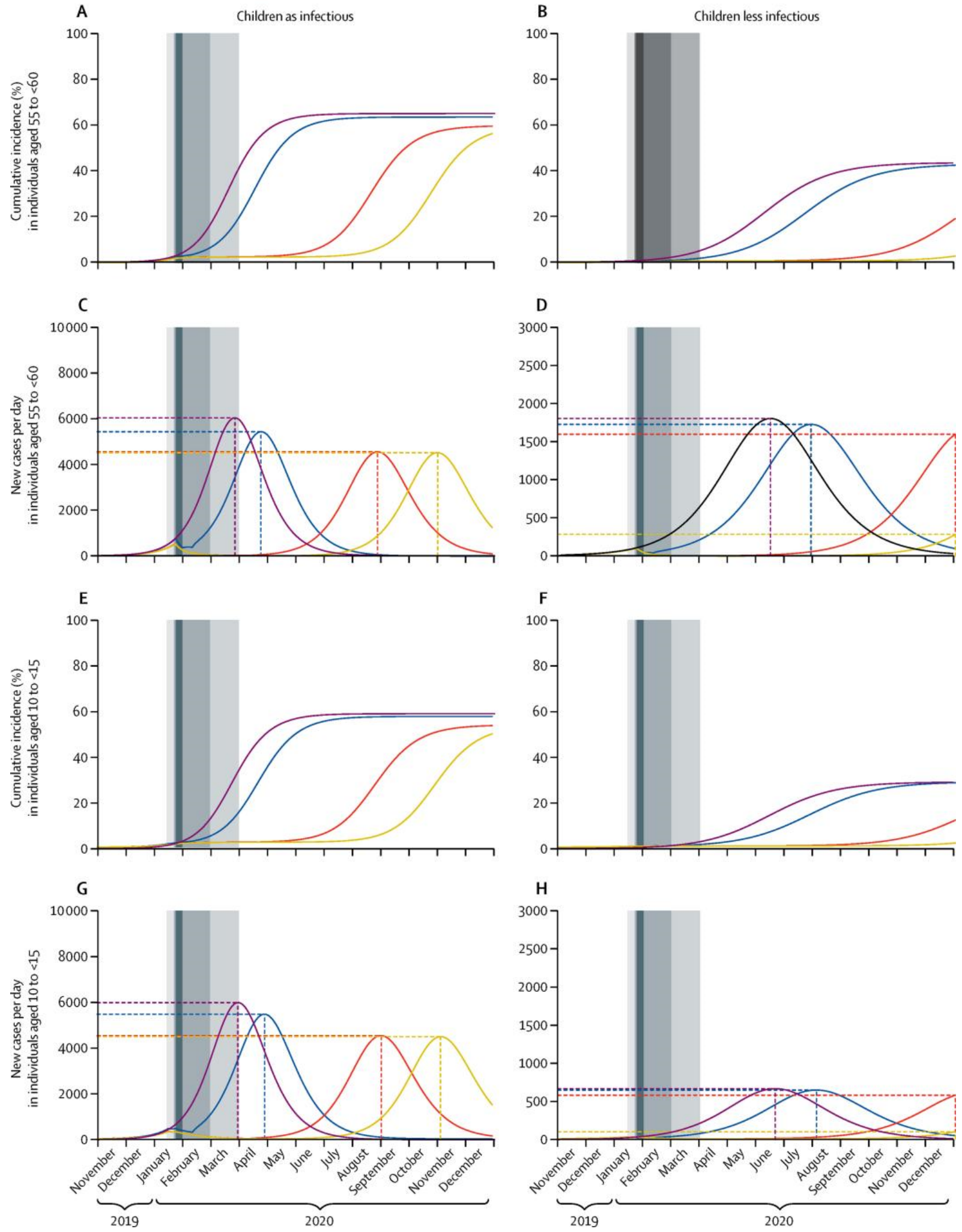

Figure 4. Effects of different intervention strategies on the cumulative incidence and new cases per day among individuals aged 55 to $<60$ years (A to D) and 10 to $<15$ years ( $\mathrm{E}$ to $\mathrm{H}$ ) from late 2019 to end-2020 [34].

Social distancing is the most effective measure to prevent uninfected people from infecting. 


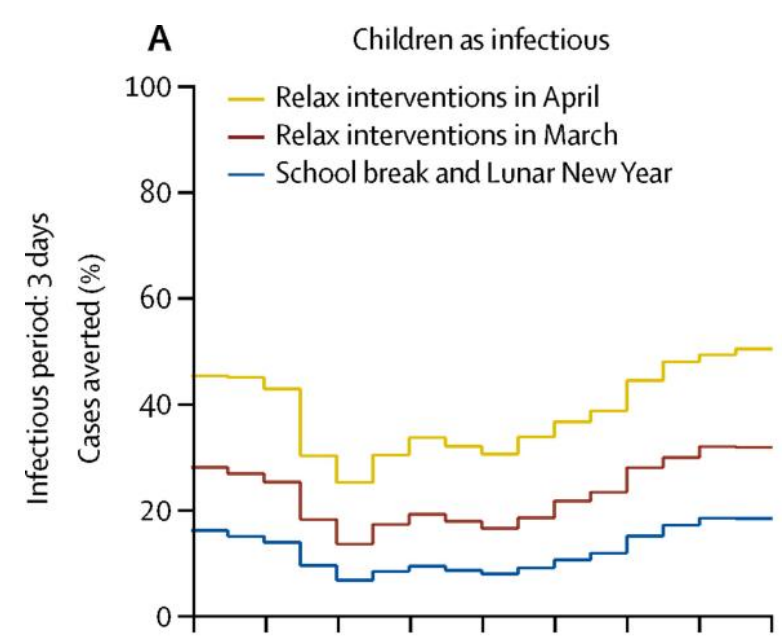

B Children less infectious
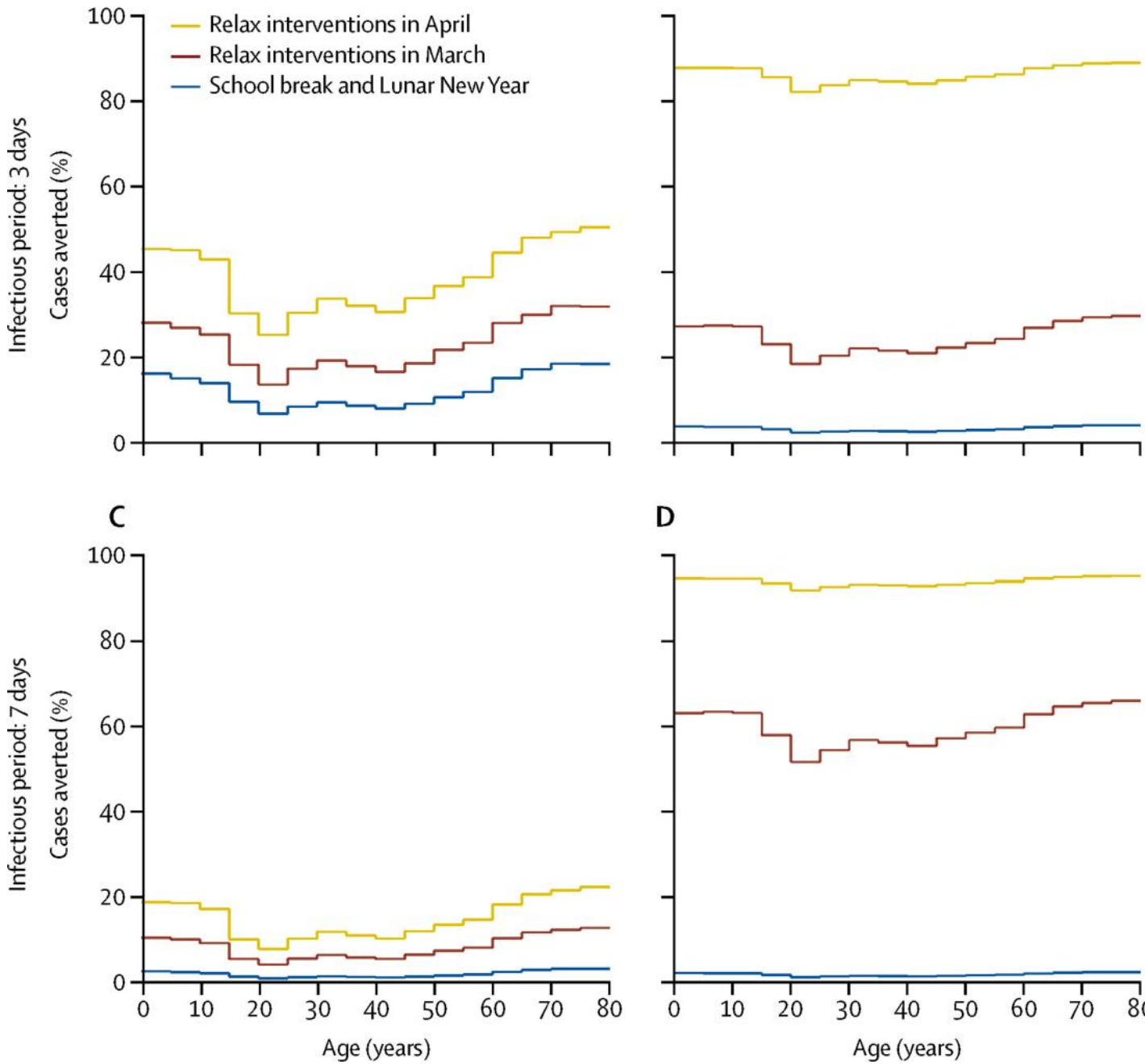

D

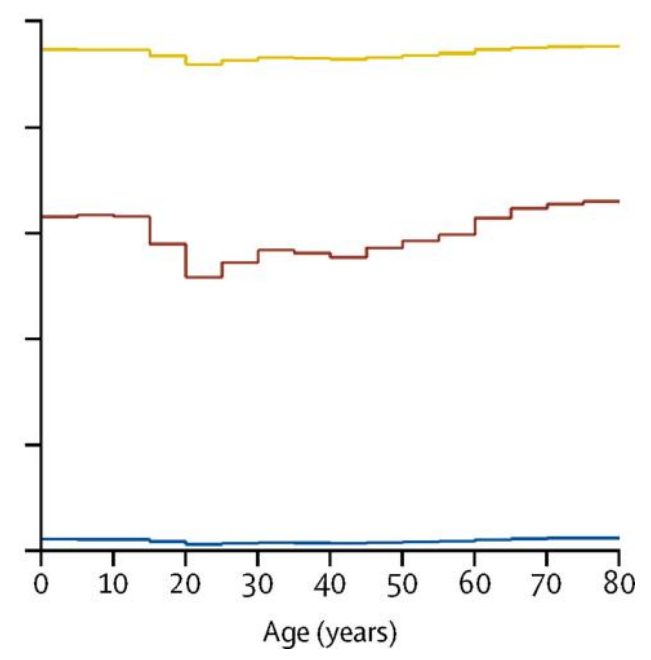

Figure 5. The modeled proportion of the number of infections averted by end-2020 by age for different physical distancing measures, assuming the duration of infectiousness to be 3 days (A, B) or 7 days (C, D) [34].

\subsection{Normalization of Epidemic Prevention and Control in Wuhan}

The normalization meant that people could basically return to normal life and work when people took protective measures. In the normal environment, people still needed to take protective measures according to the requirements of the prevention and control guidelines. Especially when people were in the places where people gathered, people needed to overcome some difficulties and insist on wearing masks; these protective measures can not be abandoned. In the normalized epidemic management in Wuhan, relevant departments mainly adhered to the principles of group prevention, group control, and precise prevention and control continuously strengthened community prevention and control and implemented grid prevention and control measures. Secondly, the relevant departments focused on returning to work and study, especially for the prevention and control guidance of medical institutions, schools, enterprises, pension places, prisons, and other key places and key institutions. They implemented various prevention and control measures such as temperature detection, health code presentation, ventilation, and disinfection. The relevant departments improved the nucleic acid detection ability of the jurisdiction as soon as possible according to the principle of timely detection, rapid disposal, accurate control, and effective treatment. They implemented that to be detected as soon as possible for the key groups. Finally, to normalize epidemic management, departments insisted on prevention. They strengthened the protection and guidance of key groups, and scientifically guided the public to wear masks according to the guidelines issued by the State Council in response to the joint prevention and control system of COVID-19.

\section{Pandemic revision- local outbreak in Beijing and Dalian}

\subsection{Pandemic in Beijing}

\subsubsection{Originality}


After 56 consecutive days without the local transmission in Beijing, the first case of COVID-19(COVID-19) was diagnosed on June 11 in a man in his 50 s with symptoms of headache, fever, and chest CT infiltration, and no contact or travel history. On the same day, the outbreak warning was triggered. In the first two cases, both have epidemiology communication to Xinfadi Agricultural wholesale market. Meanwhile, they didn't have any other locations or persons in the past 14 days. Therefore, the origin of the virus can be determined to be Xinfadi Agricultural wholesale market [32].

\section{Cases}

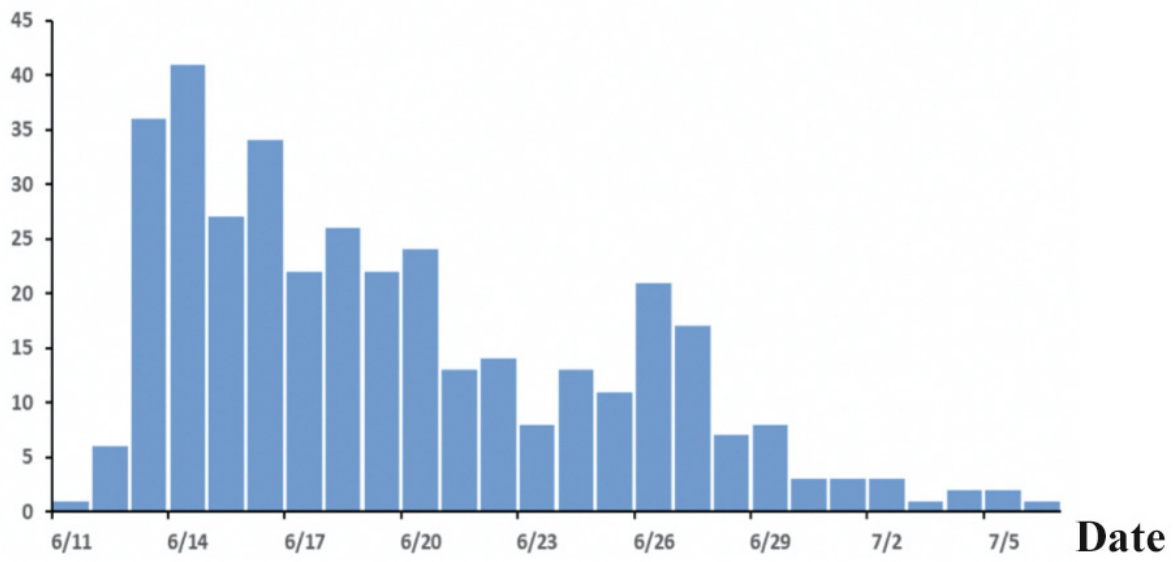

Figure 6. Number of cases in Xinfadi Market

\section{Cases}

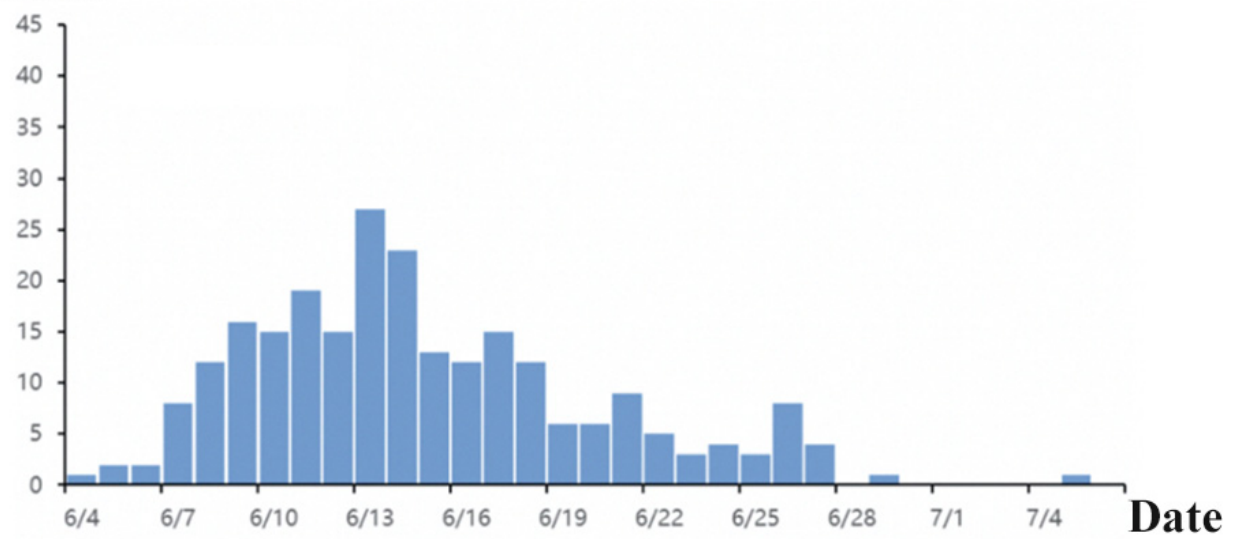

Figure 7. The number of infections in Xinfadi Market.

An epidemic distribution graph was drawn based on the onset dates of 242 confirmed cases with obvious, selfevident symptoms, showing that the earliest onset dates of cases were June 4 . The number of daily cases increased significantly from June 7 , and reached the peak on June 13 and 14, and then the number of daily cases gradually decreased. The incidence peak was mainly on June 7 , BBB 0,18 , accounting for $77.3 \%$ of all cases.

\subsubsection{New Characteristics}

The epidemiological characteristics of 368 cases of COVID-19 infection reported in the newly emerged area of Beijing were described and analyzed. It was found that this outbreak presented an outbreak pattern, covering a wide area, with obvious occupational characteristics of the infected people, and few severe or critical patients,

\subsubsection{Time-line}

The first case was reported on June 11, and the number of reported cases peaked on June 14. Since then, the number of daily reported cases has been gradually decreasing. After a small rebound on 26 and 27, the number of reported cases declined further rapidly. There were less than 5 new cases per day in the past 7 days. On 7 July, no new cases were reported for the first time. with no deaths. The age of asymptomatic infection is lower than that of confirmed cases. A higher proportion of patients are male in this outbreak, and a higher proportion is engaged in catering, food, business, and public services. This is related to the fact that the epidemic originated from the new market and the exposed population was mainly market practitioners and service workers. The cases' temporal and spatial distribution characteristics showed that the epidemic originated from the newly emerged market spread to many districts of Beijing in a short time at a fast speed, forming more epidemic spots, and $93 \%$ of the cases were infected in public places [33].

\subsubsection{Regulation Strategy}

The city has reinstated a level two public health emergency response, requiring the reporting of travel 
history to Xinfadi Market since May 30 and close contacts of anyone in Xinfadi Market requesting leave of 14 days. This isolation deprives the pathogen of its host and prevents infection. Since May 30, large-scale screening and nucleic acid tests have been carried out on those who have been to Xinfadi and those who have market contacts. Within 22 hours of the new outbreak, once the Xinfadi market was designated as a high-risk location, Beijing immediately suspended markets, closed nearby residential areas, and put employees under medical observation to curb the spread. Precise prevention and control measures have been taken, and sub-districts and towns have been classified into low, medium, and highrisk areas based on the epidemic situation. Community is the foundation

Epidemic prevention and control units, the elimination of community transmission is the primary guarantee of epidemic control. In addition to implementing epidemiological prevention measures, community-based epidemiological prevention service providers must also take care of vulnerable groups in the community, including the poor, the elderly, and the disabled, who are disproportionately affected by restricted movement measures [34].

\subsection{Pandemic in Dalian}

\subsubsection{Originality}

On July 22, 2020, COVID-19 patients were screened in the fever outpatient department of Dalian Central Hospital, Liaoning Province. On the same day, one COVID-19 patient was screened in Shengjing Hospital of Dalian Development Zone and one in Liaoyu Hospital of Dalian City. So far, after 111 days without any new local cases, another local confirmed case of COVID-19 was reported in Dalian. The Dalian Center for Disease Control and Prevention (CDC) immediately launched an emergency response and promptly launched an investigation into the outbreak. The investigation and treatment of the outbreak are now reported to provide a reference for the prevention and control of COVID-19. The outbreak began in a frozen seafood processing workshop in Dalian City, where contaminated seafood from abroad entered the workshop and infected a few workers. It then spread rapidly among workers in the workshop and spread to their living communities [35].

\subsubsection{Time-line}

The cases of this outbreak were mainly concentrated in the first 5 days (22-26 July), with 70 cases reported, accounting for $59.32 \%$ of all cases.

An epidemic curve of 92 confirmed cases was drawn based on the epidemiological investigation records and the case information from the Infectious Disease Report Management Information System (the confirmed cases converted from asymptomatic infected persons to confirmed cases and the confirmed cases without an onset date in the epidemiological investigation report were counted according to the diagnosis date). The earliest onset of the cases was July 9, followed by 6 cases on July 18 , followed by a significant increase in the number of daily cases, which peaked on July 23 ( 7 cases). Then the number of daily cases fluctuated. The incidence peak (2131 July) accounted for $72.03 \%(85 / 118)$ of the total cases [35].

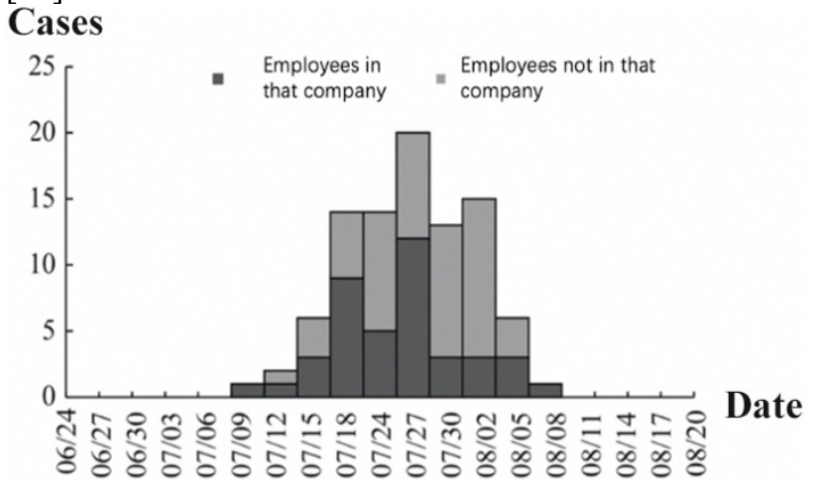

Figure 8. Infection Distribution in Dalian.

\subsubsection{New characteristics}

Population distribution Among the 118 cases, there were 58 males and 60 females, with a male-to-female sex ratio of $0.97: 1$. The cases ranged from 1 month to 73 years, with a mean age of 43 years. Among the 92 confirmed cases, 41 were male, and 51 were female, with a male-to-female ratio of $0.81: 1$. Among the 26 asymptomatic infected patients, 17 were males, and 9 were females, and the male-to-female ratio was $1.89: 1$.

Among the 118 cases, 69 were workers, accounting for $58.4 \%$; Then there were domestic and unemployed workers (16 cases, 13.5\%), cadres and staff ( 9 cases, $7.6 \%$ ), students ( 5 cases, $4.2 \%$ ), business service workers ( 4 cases, 3.3\%), and migrant workers (4 cases, 3.3\%). 3 quit, accounting for $2.5 \%$; Catering and food industry personnel, drivers, farmers every 2 cases, accounting for $1.6 \% ; 1$ case for children and 1 case for medical personnel, accounting for $0.8 \%$ respectively. Among them, 56 cases were employees of a food company, 46 cases were employees of the production workshop, and 10 cases were employees of other departments.

Regional distribution Of the 118 cases, 96 were living in the Ganjingzi District, of which 71 were confirmed, and 25 were asymptomatic infected. Of the 17 cases living in Jinpu New District, 16 were confirmed, and 1 was asymptomatic infected. The number of confirmed cases living in Pulandian District, Xigang District, and Wafangdian City is 3, 1, and 1. Among them, 96 cases in Ganjingzi District were all from Dalian Bay Street [35].

\subsubsection{Regulation Strategy}

After the epidemic outbreak on July 22, 2020, Dalian City acted quickly and decisively to stop the transmission chain. The employees and family members of a food company with cases were quarantined as soon as possible. While extensive nucleic acid testing was carried out for all staff, close contacts were defined, and the scope of 
screening and control of close contacts was further expanded. For frozen flow product storage, processing, and sales potential with the spread of the virus amplification of high-risk places, such as strengthening regulations and daily control, targeted prevention and control measures, such as improving health conditions, shorten the continuous working time, keep the interpersonal distance, completes the individual occupational protection, employee's health monitoring. It is effective to reduce the spread risk of a novel coronavirus in such places.

\section{Comparison, Characteristics, Regulation, and Referential experiences from these pandemics+}

\subsection{Overview: disease in 2019}

COVID-19 had become the most in-depth experience for many people to come by the year 2020. COVID-19 had brought distribution and fear to our lives. At the beginning phase of COVID-19 (SARS-CoV-2) in Wuhan, China, released the genomic sequence of the virus on Jan 10, 2020, and began enacting a raft of rigorous countermeasures later in the same month. After the first reported cases of the disease, Wuhan was placed under a strict lockdown that lasted 76 days. From June to August, COVID-19 incidence was highest in persons aged 20 to 29 years, who accounted for lower than $20 \%$ of all confirmed cases. The southern United States experienced regional outbreaks of COVID-19 in June. As of September 21, 2020, the COVID-19 pandemic had resulted in more than $6,800,000$ reported U.S. cases and more than 199,000 associated deaths. Early in the pandemic, COVID-19 incidence was highest among older adults. [36] Recently, the CDC study provides strong evidence that mRNA COVID-19 vaccines, including the Pfizer-BioNTech and Moderna vaccines, are highly effective in preventing SARS-CoV-2 infections in realworld conditions. And the CDC study showed that, once fully vaccinated, a person's risk of infection is reduced by up to $90 \%$. The efficacy seen in clinical trials is now being shown in the real world [37].

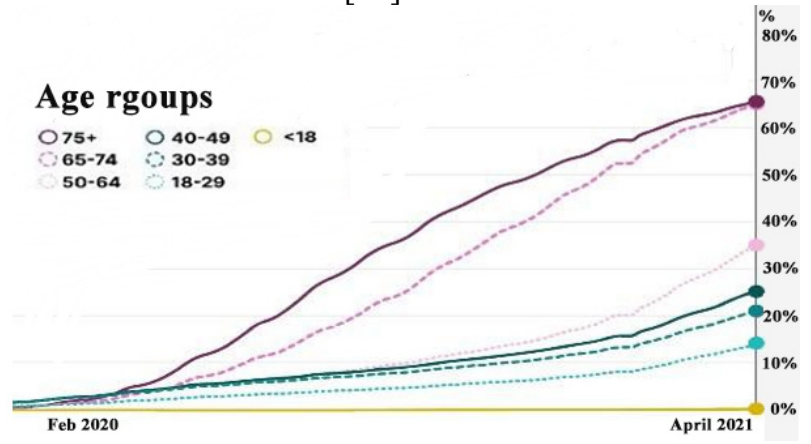

Figure 9. Percent of People Fully Vaccinated [38]

Figure 9 shows that the population that has been fully vaccinated is growing over time [38]. Because by now, the major countries in the world had invented the vaccine for COVID-19. More and more populations were being vaccinated with different types like Pfizer-BioNTech, Moderna, and Johnson \& Johnson's Janssen. The different types of vaccines had different requirements for the patient, some vaccines required two total shots to be fully vaccinated, and some only took one.

Figure $\mathbf{1 0}$ shows the number of COVID-19 vaccination within a given population. The total number of people vaccinated, depending on the specific dose regime [39].

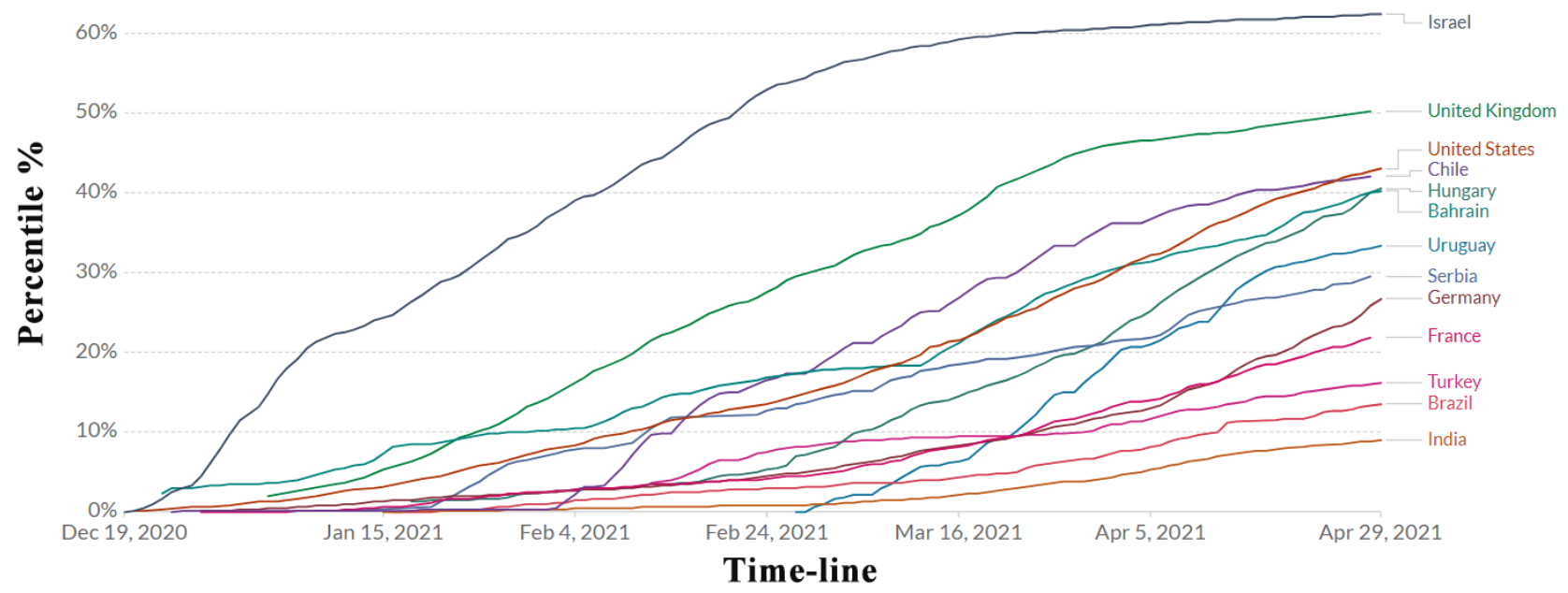

Figure 10. People Who Received at least one dose of COVID-19 vaccine [39].

\subsection{Characteristics of COVID-19}

The outbreak of COVID-19 in China peaked in February, with an average rate of more than 3,000 newly confirmed cases per day. Even worse, the international spread of COVID-19 increased from late February. The infections have been reported from an increasing number of countries [40]. And with the time of more than a year after the first cases of COVID-19 were detected, the scientists have turned to look at the genes of patients with severe COVID19 to understand if their bodies mount an immune defense differently healthy patients do. Since the first cases of COVID-19 were associated with direct contact with the South China Seafood Wholesale Market in Wuhan, it is speculated that human-to-animal transmission is the main mechanism. Subsequent cases are not associated with this mechanism of exposure. Therefore, we believe that the 
virus is also capable of human-to-human transmission, with symptomatic people being the most common source of COVID-19 transmission. The possibility of transmission before symptoms appear appears to be small, but it cannot be ruled out. In addition, it has been suggested that asymptomatic individuals may transmit the virus. In January, US researchers set an ambitious goal of developing a vaccine against the coronavirus within 12 to 18 months. That would be a world record. The mumps vaccine is considered the fastest vaccine, taking four years from scientific concept to approval in 1967. The search for an HIV vaccine has been going on for 36 years. As of December 12, 2020, and December 19, 2020, safety monitoring of these vaccines has been the most intense and comprehensive in the history of the United States, using the Vaccine Adverse Event Reporting System (VAERs), the Voluntary Reporting System, and V Safety to implement a system in the initial phase of the COVID-19 National Vaccination Program [41]. Two COVID-19 vaccines (Pfizer Biotech and Moderna) have received emergency authorization from the U.S. Food and Drug Administration for use. Two doses of both vaccines are required for the full series. Recommended dose interval: 21 days for Pfizer-Biotech and 28 days for Moderna; however, doses may be spaced up to 42 days apart when delays are unavoidable. Two analyses of COVID-19 vaccination data were performed for people who initiated a series of vaccinations on December 14, 2020, solstice, between February 14, 2021, and those who reported to the CDC on February 20, 2021. The first analysis was to determine whether people who received the first dose had enough time to receive the second dose [42]. In the current Severe Acute Respiratory Syndrome (SARS-CoV-2) pandemic, laboratory diagnosis is critical for clinical care and informs the public health response. The first molecular test protocol was launched on January 13, 2020, and was quickly shared. The program focuses on enveloped and RNA-dependent RNA polymerase gene targets. By the end of January 2020, reports from European laboratories indicate that batches of commercially customized primers and probes for SARS-CoV-2 testing may be used for synthetic control material contamination of $\mathrm{E}$ gene targets. This observation was published on February 5, 2020, in the Emerging Viral Disease Specialist Laboratory Network (LabNet). An appeal for more detailed information was launched to assess the seriousness of the situation. Timeline and extent of product and molecular diagnostics of contamination problems in 10 European laboratories during laboratory delays to COVID-19. Others reported sporadic pollution. Drastic changes in pollution levels; The reported cycle thresholds range from 23 to 39 . These laboratories systematically excluded other potential contaminants associated with their laboratories. A vaccine to prevent SARS-CoV-2 infection was diagnosed, and 161 cases of PCR-confirmed infection were identified. Within 13 days of the first or second dose of vaccine, the immune status of 67,483 people per day was considered inconclusive, of which 33 PCR-confirmed infections were excluded from the results. Two sources of partial immunization days were reported. Among those who did not receive a second dose of vaccine during the study period, 5 PCR-confirmed infections were reported within
15,868 person-days approximately 14 days after the first dose. Three PCR-confirmed infections were reported within approximately 14 days after the first dose of vaccine and during the 25,988 person-days after receiving the second dose. Altogether, these are eight PCR-confirmed infections that occurred during the 41,856 person-days of partial immunization. Three PCR-confirmed infections occurred during the 78,902 person-days of full immunization.

\section{Conclusion}

At the start of COVID-19, epidemiological properties are not widely known. New studies and publications will take a month to a year to make it important to know and understand the level of knowledge of health care personnel and the management of preparedness affecting patients with the virus. The COVID-19 pandemic affected the world in many ways. The lack of information, the need for accurate information, and the speed with which it is disseminated are all important because this epidemic requires the cooperation of the entire population. Our rapid survey has responded well, showing that health care providers and the public are well informed about the coronavirus. They recognized the measures that needed to be taken to reduce the spread of the disease. Based on the available knowledge, the public gets a lot of information from social media. The medical community and governments need to develop strategies to ensure that accurate information needs to be disseminated on these forums. Public awareness is quite high, and knowledge of communication channels must be made a top priority throughout the pandemic.

\section{References}

1. Du Toit, A., Outbreak of a novel coronavirus. Nat Rev Microbiol, 2020. 18(3): p. 123.

2. Huang, C., et al., Clinical features of patients infected with 2019 novel coronavirus in Wuhan, China. Lancet, 2020. 395(10223): p. 497-506.

3. Full Text: Fighting COVID-19: China in Action. 2020.

4. COVID-19 CORONAVIRUS PANDEMIC. 2021 [cited $2021 \quad 17$ May]; Available from: https://www.worldometers.info/coronavirus/. .

5. Callaway, E. and D. Cyranoski, China coronavirus: Six questions scientists are asking. Nature, 2020. 577(7792): p. 605-607.

6. Li, W., et al., Characteristics of Household Transmission of COVID-19. Clin Infect Dis, 2020. 71(8): p. 1943-1946.

7. Coronavirus disease (COVID-19): How is it transmitted? 2021; Available from: https://www.who.int/emergencies/diseases/novelcoronavirus-2019/question-and-answers-hub/q-adetail/coronavirus-disease-covid-19-how-is-ittransmitted.

8. Rothe, C., et al., Transmission of 2019-nCoV Infection from an Asymptomatic Contact in Germany. N Engl J 
Med, 2020. 382(10): p. 970-971.

9. Symptoms of Coronavirus.

10. Carly Eastin, T.E., Clinical Characteristics of Coronavirus Disease 2019 in China

Guan W, Ni Z, Hu Y, et al. N Engl J Med. 2020 Feb 28 [Online ahead of print] DOI: 10.1056/NEJMoa2002032. The Journal of Emergency Medicine, 2020. 58(4): p. 711-712.

11. Coronavirus. . Available from: https://www.who.int/healthtopics/coronavirus\#tab=tab_1.

12. Who is at high risk from coronavirus (clinically extremely vulnerable. 2021.

13. Fang, L., G. Karakiulakis, and M. Roth, Are patients with hypertension and diabetes mellitus at increased risk for COVID-19 infection? Lancet Respir Med, 2020. 8(4): p. e21.

14. Hutchins, S.S., et al., Protection of racial/ethnic minority populations during an influenza pandemic. Am J Public Health, 2009. 99 Suppl 2(Suppl 2): p. S261-70.

15. Woo, P.C., et al., Coronavirus diversity, phylogeny and interspecies jumping. Exp Biol Med (Maywood), 2009. 234(10): p. 1117-27.

16. Cheng, V.C., et al., Severe acute respiratory syndrome coronavirus as an agent of emerging and reemerging infection. Clin Microbiol Rev, 2007. 20(4): p. 660-94.

17. Zaki, A.M., et al., Isolation of a novel coronavirus from a man with pneumonia in Saudi Arabia. N Engl J Med, 2012. 367(19): p. 1814-20.

18. Chan, J.F., et al., Middle East respiratory syndrome coronavirus: another zoonotic betacoronavirus causing SARS-like disease. Clin Microbiol Rev, 2015. 28(2): p. 465-522.

19. He, F., Y. Deng, and W. Li, Coronavirus disease 2019: What we know? J Med Virol, 2020. 92(7): p. 719-725.

20. Lu, R., et al., Genomic characterisation and epidemiology of 2019 novel coronavirus: implications for virus origins and receptor binding. Lancet, 2020. 395(10224): p. 565-574.

21. Covid19. Available from: https://clinicaltrials.gov/ct2/results? cond $=$ Covid19\& Search $=$ Apply\&age $\_\mathrm{v}=$ \&gndr $=$ \&type $=\& \mathrm{rslt}=$.

22. Wei, P.-F., Diagnosis and Treatment Protocol for Novel Coronavirus Pneumonia (Trial Version 7). Chinese Medical Journal, 2020. 133(9): p. 1087-1095.

23. Yang, L.M., et al., Cloning and characterization of a novel feline IFN-omega. J Interferon Cytokine Res, 2007. 27(2): p. 119-27.

24. Lopinavir. Available from: https://druginfo.nlm.nih.gov/m.drugportal/name/lopi navir.

25. Beigel, J.H., et al., Remdesivir for the Treatment of Covid-19 - Final Report. N Engl J Med, 2020. 383(19): p. 1813-1826.

26. Sheahan, T.P., et al., Comparative therapeutic efficacy of remdesivir and combination lopinavir, ritonavir, and interferon beta against MERS-CoV. Nat Commun, 2020. 11(1): p. 222.

27. Ji, S., et al., Unique synergistic antiviral effects of Shufeng Jiedu Capsule and oseltamivir in influenza A viral-induced acute exacerbation of chronic obstructive pulmonary disease. Biomed Pharmacother, 2020. 121: p. 109652.

28. COVID-19 Vaccines. 2021; Available from: https://www.who.int/emergencies/diseases/novelcoronavirus-2019/covid-19-vaccines.

29. Thanh Le, T., et al., The COVID-19 vaccine development landscape. Nat Rev Drug Discov, 2020. 19(5): p. 305-306.

30. Jonathan Corum, C.Z., How the Sinovac Vaccine Works. The New York Times, 2021.

31. Park, K.S., et al., Non-viral COVID-19 vaccine delivery systems. Adv Drug Deliv Rev, 2021. 169: p. 137-151.

32. Wu, Z., et al., Time Course of a Second Outbreak of COVID-19 in Beijing, China, June-July 2020. JAMA, 2020. 324(14): p. 1458-1459.

33. Zhang, Y., et al., [Epidemiological characteristics of COVID-19 cases in outbreak in Xinfadi market in Beijing]. Zhonghua Liu Xing Bing Xue Za Zhi, 2021. 42(3): p. 1-5.

34. Wang, X.Y., Y.Q. Zhang, and L.W. Cai, Spatiotemporal characteristics of the COVID-19 resurgence in the metropolitan wholesale market of Beijing, China. J Travel Med, 2021. 28(2).

35. <Epidemiological investigation of COVID-19 outbreak in Dalian, Liaoning Province from July to August, 2020 .pdf>.

36. Tegan K. Boehmer, PhD; Jourdan DeVies, MS; Elise Caruso, MPH; Katharina L. van Santen, MSPH; Shichao Tang, PhD; Carla L. Black, PhD; Kathleen P. Hartnett, PhD; Aaron Kite-Powell, MS; Stephanie Dietz, PhD; Matthew Lozier, PhD; Adi V. Gundlapalli, MD, PhD Changing Age Distribution of the COVID19 Pandemic - United States MMWR Morb Mortal Wkly, vol, no, pp, (2020). https://pubmed.ncbi.nlm.nih.gov/33001872/

37. Covid Data Tracker Weekly Review Center of Disease Control and Prevention (2021) https://www.cdc.gov/coronavirus/2019-ncov/coviddata/covidview/index.html

38. Covid Data Tracker Weekly Review Center of Disease Control and Prevention (2021). https://www.cdc.gov/coronavirus/2019-ncov/coviddata/covidview/

39. Hannah Ritchie, Esteban Ortiz-Ospina, Diana Beltekian, Edouard Mathieu, Joe Hasell, Bobbie Macdonald, Charlie Giattino, Cameron Appel, Max Roser Statistics and Research Coronavirus (COVID19) Vaccinations Our World In Data, vol, no, pp, (2021). https://ourworldindata.org/covid-vaccinations

40. Jennifer L. Kriss, PhD1; Laura E. Reynolds, MPH1; Alice Wang, PhD1; Shannon Stokley, DrPH1; Matthew M. Cole, MPH1; LaTreace Q. Harris, MPH1; 
Lauren K. Shaw, MS1; Carla L. Black, PhD1; James A. Singleton, PhD1; David L. Fitter, MD1; Dale A. Rose, PhD1; Matthew D. Ritchey, DPT1; Robin L. Toblin, PhD1 COVID-19 Vaccine Second-Dose Completion and Interval Between First and Second Doses Among Vaccinated Persons International Committee of Medical Journal, vol 70, no 11, pp 389395

https://www.cdc.gov/mmwr/volumes/70/wr/mm7011 e2.htm

41. Ben $\mathrm{Hu}$, Hua Guo, Peng Zhou \& Zheng-Li Shi Characteristics of SARS-CoV-2 and COVID-19 Nature Reviews Microbiology, vol 19, no, pp 141154(2020). https://www.nature.com/articles/s41579020-00459-7

42. Julianne Gee1; Paige Marquez1; John Su1; Geoffrey M. Calvert1; Ruiling Liu1; Tanya Myers1; Narayan Nair2; Stacey Martin1; Thomas Clark1; Lauri Markowitz1; Nicole Lindsey1; Bicheng Zhang1; Charles Licata1; Amelia Jazwa1; Mark Sotir1; Tom Shimabukuro1 First Month of COVID-19 Vaccine Safety Monitoring — United States International Committee of Medical Journal, vol 70, no 8, pp 283288 https://www.cdc.gov/mmwr/volumes/70/wr/mm7008 e3.htm 